\title{
Assessment of thick-film resistors for manufacturing piezoresistive sensors
}

Thomas Maeder, Caroline Jacq and Peter Ryser

École Polytechnique Fédérale de Lausanne (EPFL), Switzerland
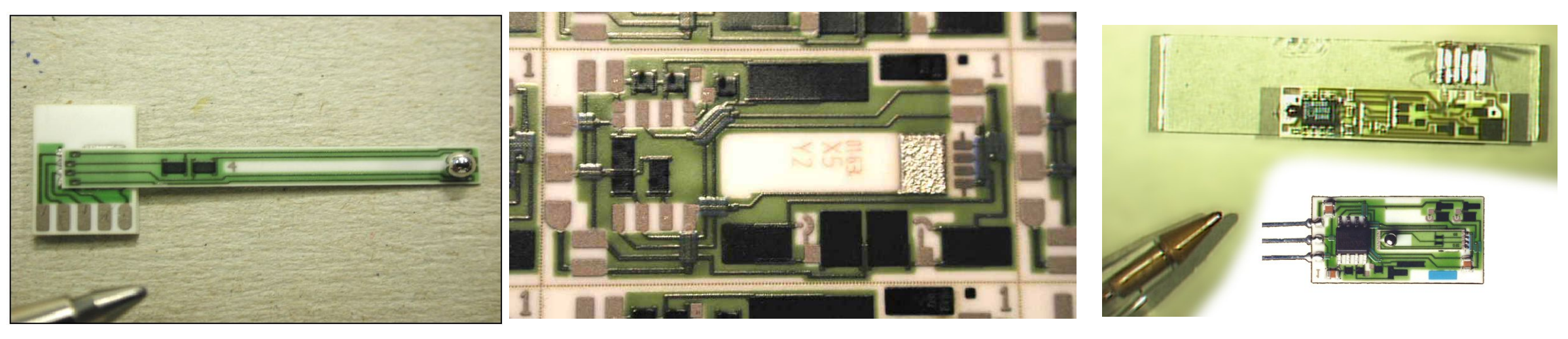


\section{Outline}

1. Introduction - manufacturing \& trimming issues

2. Resistor study

3. Overglazing, trimming, etc.

4. Conclusions \& outlook 


\section{Outline}

1. Introduction - manufacturing \& trimming issues

2. Resistor study

3. Overglazing, trimming, etc.

4. Conclusions \& outlook 


\section{Typical thick-film piezoresistive sensor}

- Typical elements

- Sensing bridge

- Offset trim

- TCO trim

- Differential amplifier

- Typical values ( \pm )

- Offset $230 \mathrm{mV} / \mathrm{V}$

- Response $\sim 3 \mathrm{mV} / \mathrm{V}$

- $\mathrm{TCO} \sim 1 \mu \mathrm{V} / \mathrm{V} / \mathrm{K}$ $(50 \mathrm{~K}: \sim 0.05 \mathrm{mV} / \mathrm{V})$

- For 0.1\% F.S.:

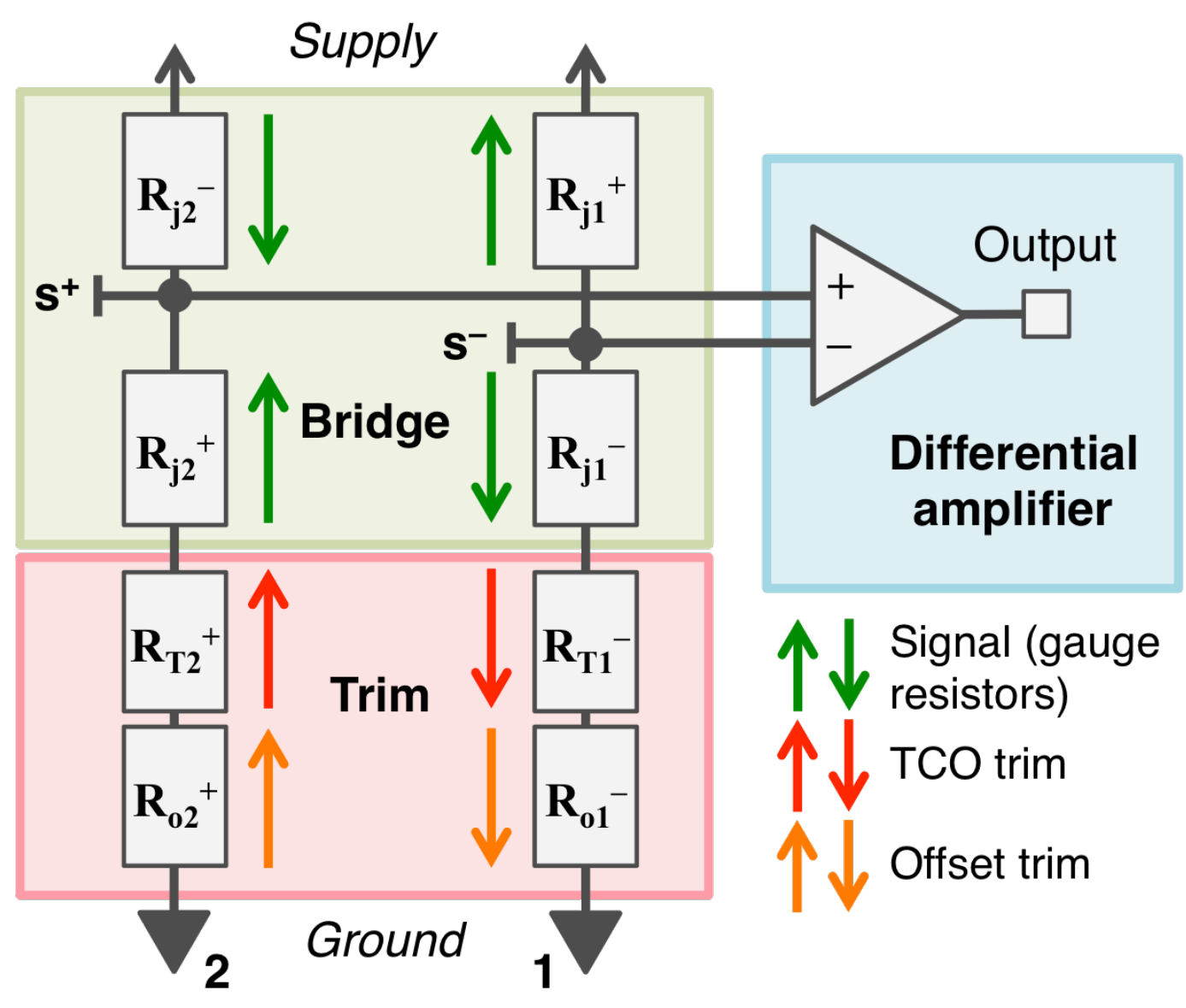

- Offset reduction $\sim 101000 x$

- Stability (bridge) 10 ppm 


\section{Why trim?}

\section{- Modern digital chips}

- Input stage usually PGA (programmable-gain amplifier)

- Gain limited by signal

- In raw state, offset dominates signal, >> response

- For optimal use, reduce offset to < response

- With typical raw offset $\sim 30 \mathrm{mV} / \mathrm{V}$, max. gain $\sim 30 \mathrm{x}$

- With typical response $\sim 3 \mathrm{mV} / \mathrm{V}$, typ. gain required $\sim 200 x$

- Reduce offset typically by $\sim 10 . . .30 x$

- Trimming of TCO usually not necessary with chips

- Typically, temperature error $<10 \%$ of piezoresistive response

- Can be done digitally

- Laser trim: large-scale production; better temperature sensing 


\section{Examples - pressure cell}

\section{Ceramic: classical layout}

- All-active bridge

- Coarse offset trim on cell

- Direct TCO trim

- Need good amplifier - usually not accessible after mounting of electronics

\section{Steel: changes}

- Issue: trim on dielectric

- Coarse offset trim off-cell

- Indirect TCO trim

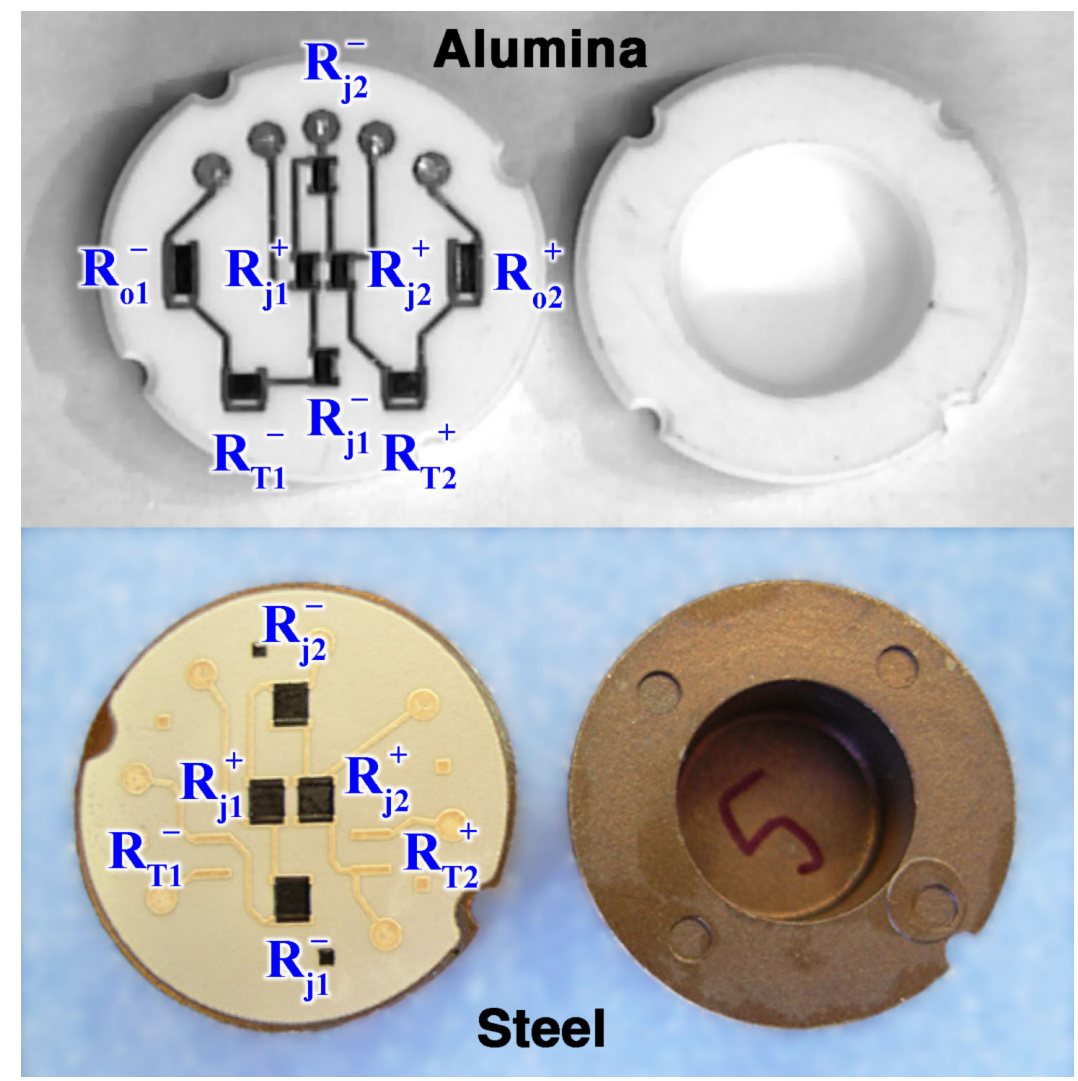

- PTC resistor on cell

- Normal resistor in parallel 


\section{Examples - cantilever force cell}

- All-active bridge

- Discrete offset trim (stable, active, no TCO change)

- Coarse classical trim (more precise)

- No TCO trim (on base, with fine trim)

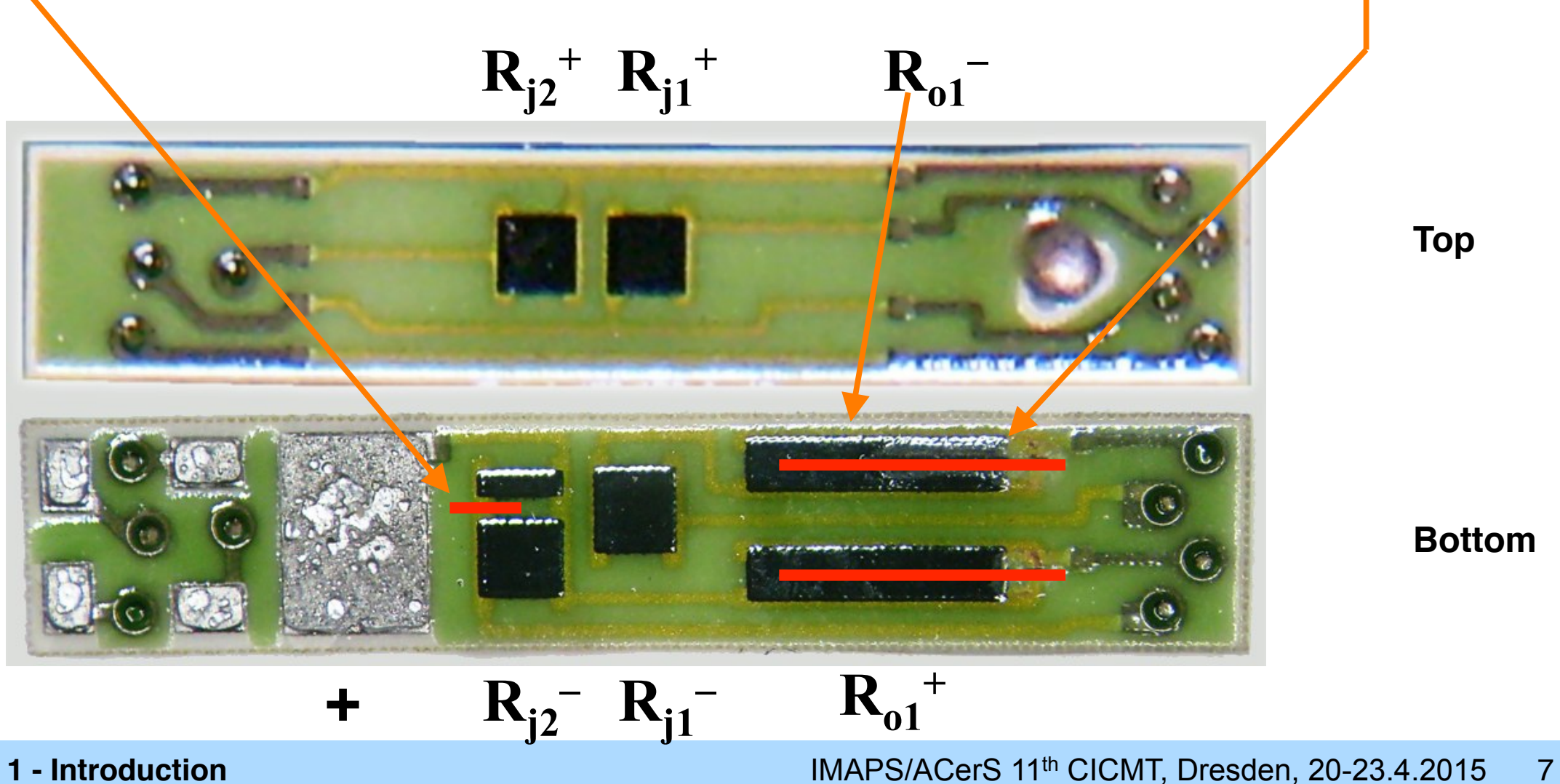




\section{Examples - glass-sealed pressure cell}

- All-active bridge

- Discrete offset trim cuts only on cell

- All other trims on separate module
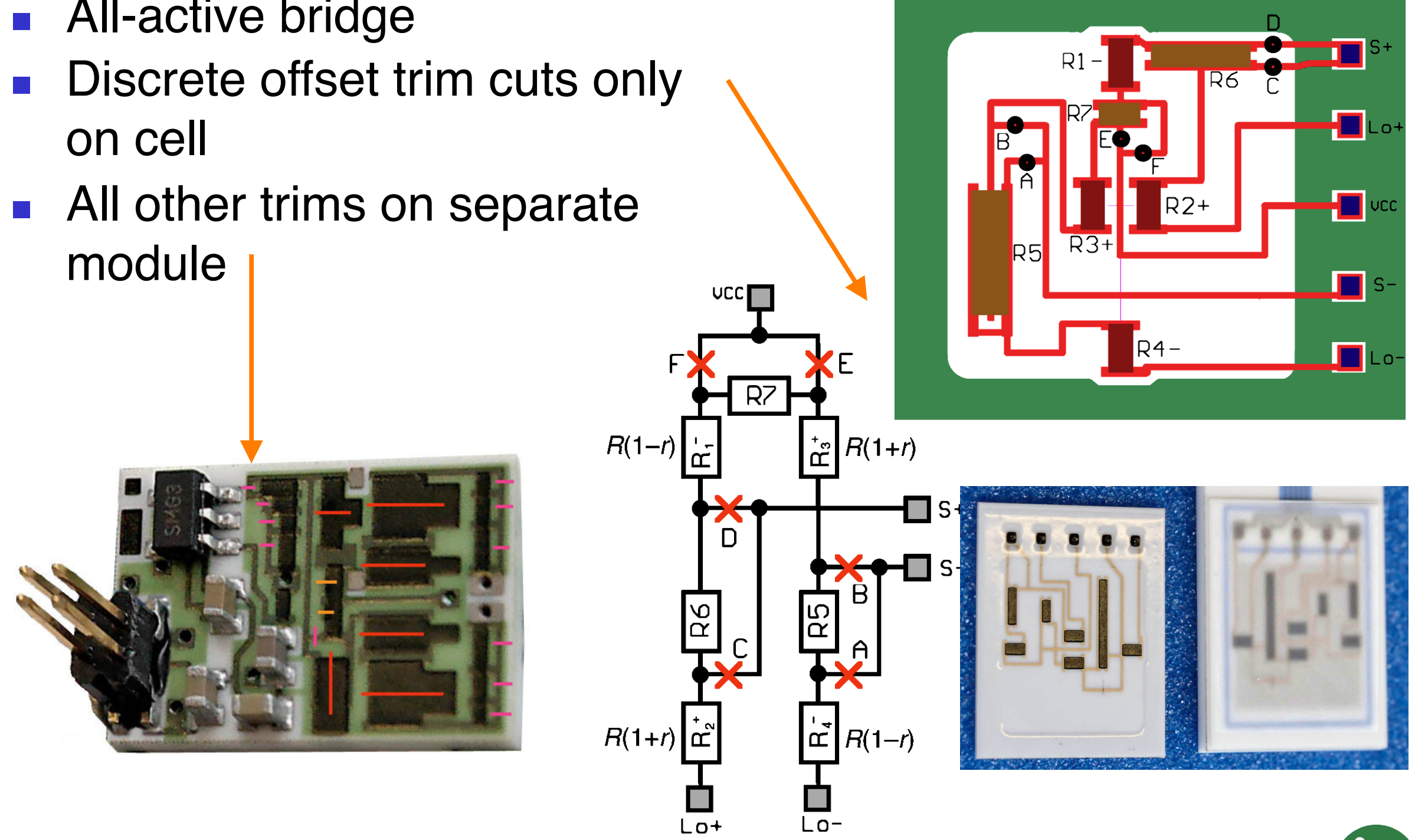

$\mathrm{LO}^{+} \mathrm{LO}$ 


\section{Trimming of sensor electronics}

- Normally passive \& active part

- High resistor values often problematic

- Harsh post-processing (breaking, soldering, ultrasound, ...)
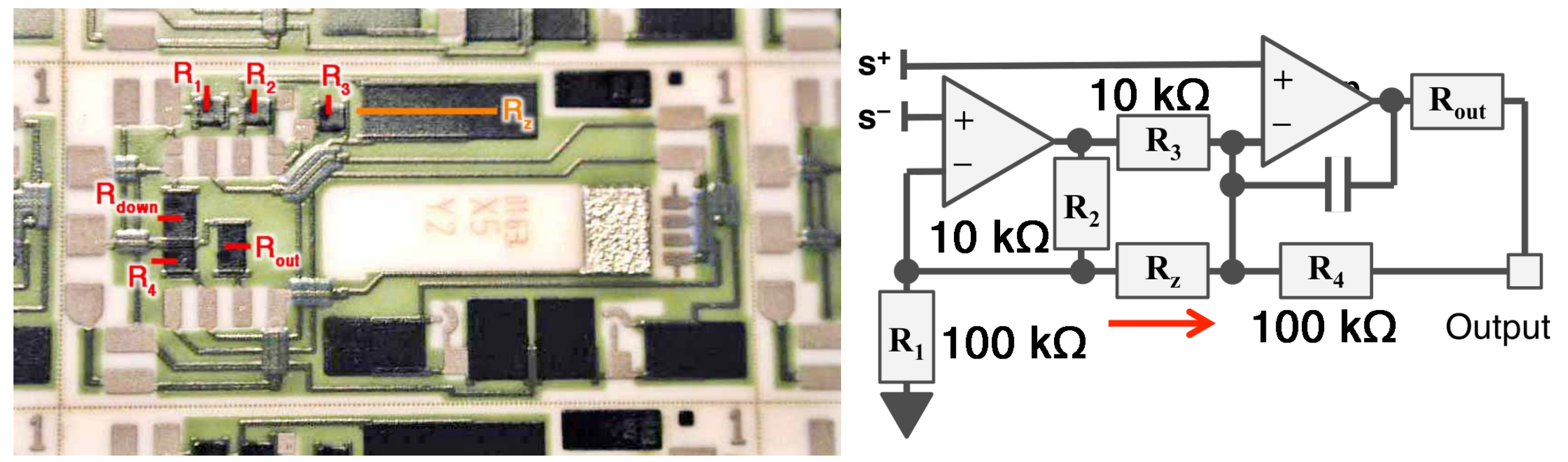


\section{Factors for offset, TCO \& stability}

- Resistor interactions

- Substrate $\left(\mathrm{Al}_{2} \mathrm{O}_{3}\right.$, dielectric, LTCC...)

- Terminations

- Overglaze

- TCO $\neq$ TCR; TCO determined by TCR tracking

- Trimming

- Discrete (stable) or classical (precise)

- Trimming resistor used (coarse: use same as bridge)

- Terminations (material near terminations $\neq$ away)

- Parameters \& resistor material

- Post-processing 


\section{Outline}

1. Introduction - manufacturing \& trimming issues

\section{Resistor study}

3. Overglazing, trimming, etc.

4. Conclusions \& outlook 


\section{Resistor study}

\begin{tabular}{|c|c|c|c|}
\hline No & $\begin{array}{l}\text { Film } \\
\text { (Sheet res.) } \\
\text { Screen }^{\S}\end{array}$ & Composition & \\
\hline 1 & $\begin{array}{l}\text { Conductor } \\
325 / 40\end{array}$ & $\begin{array}{l}\text { A) ESL } 9635 \mathrm{G}^{\dagger} \\
\text { B) ESL } 9635 \mathrm{~B}^{\dagger} \\
\text { C) DP } 5104^{\dagger} \\
\text { D) ESL } 8837^{*} \\
\text { E) ESL } 9695^{\#} \\
\text { F) ESL } 9562 \mathrm{G}^{\#} \\
\text { G) ESL } 9912 \mathrm{~K}^{\# \#}\end{array}$ & $\begin{array}{l}(\mathrm{Pb}) \\
(\mathrm{Pb}) \\
(\mathrm{Pb}, \mathrm{Cd}) \\
(\mathrm{Pb})\end{array}$ \\
\hline \multirow{3}{*}{2} & $\begin{array}{l}\text { Resistor } \\
(100 \Omega \text { PTC }) \\
325 / 40\end{array}$ & K) ESL 2612I & $(\mathrm{Pb})$ \\
\hline & $\begin{array}{l}\text { Resistor } \\
(100 \Omega) \\
325 / 40 \\
\end{array}$ & $\begin{array}{l}\text { M) DP } 2021 \\
\text { N) ESL R312P } \\
\text { O) ESL } 3912 \\
\end{array}$ & $\begin{array}{l}(\mathrm{Pb}) \\
(\mathrm{Pb}) \\
(\mathrm{Pb}, \mathrm{Cd})\end{array}$ \\
\hline & $\begin{array}{l}\text { Resistor } \\
(10 \mathrm{k} \Omega) \\
325 / 40\end{array}$ & $\begin{array}{l}\text { Q) DP } 2041 \\
\text { S) ESL R314P } \\
\text { T) ESL } 3984 \\
\text { U) ESL } 3914\end{array}$ & $\begin{array}{l}(\mathrm{Pb}) \\
(\mathrm{Pb}) \\
(\mathrm{Pb}, \mathrm{Cd}) \\
(\mathrm{Pb}, \mathrm{Cd})\end{array}$ \\
\hline 3 & $\begin{array}{l}\text { Overglaze } \\
325 / 20\end{array}$ & $\begin{array}{l}\text { V) ESL G-485-1 }{ }^{\mathrm{a}} \\
\text { W) ESL G-481 }{ }^{\mathrm{a}} \\
\text { X) ESL } 4771 \mathrm{P}^{\mathrm{b}} \\
\text { Z) DP QQ600 }\end{array}$ & $\begin{array}{l}(\mathrm{Pb}) \\
(\mathrm{Pb})\end{array}$ \\
\hline
\end{tabular}

- (Substrate = alumina)

- Termination material

- Resistor material \& length

- Overglaze material

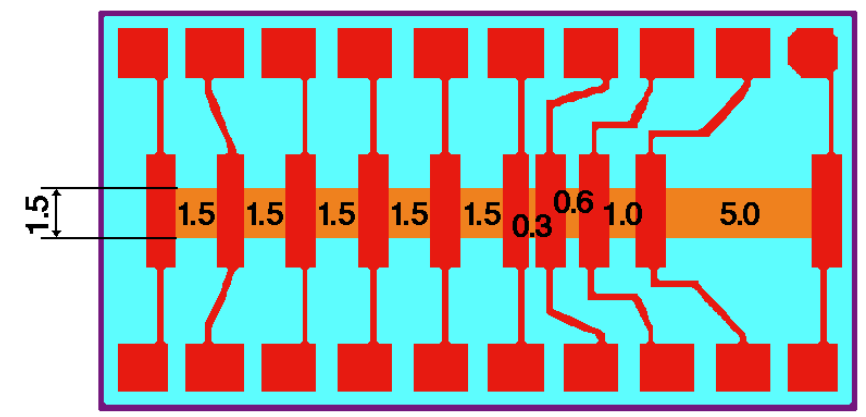

$\mathrm{ESL}=$ Electroscience Laboratories $\mathrm{DP}=\mathrm{DuPont}$ 


\section{Processing parameters}

- Resistor under...overfired

- See whether this changes its interactions with overglaze

- Overglaze under...overfired

- Extent of effect on resistor

\begin{tabular}{|c|c|c|c|}
\hline Code & Conductor & Resistor & Overglaze \\
\hline - - & $850^{\circ} \mathrm{C}(\mathrm{n})$ & $825^{\circ} \mathrm{C}\left(\mathrm{n}-25^{\circ} \mathrm{C}\right)$ & $\begin{array}{ll}\text { VWZ: } & 575^{\circ} \mathrm{C}\left(\mathrm{n}-25^{\circ} \mathrm{C}\right) \\
\mathrm{X}: & 525^{\circ} \mathrm{C}\left(\mathrm{n}-25^{\circ} \mathrm{C}\right)\end{array}$ \\
\hline $\mathrm{n}$ - & $850^{\circ} \mathrm{C}(\mathrm{n})$ & $850^{\circ} \mathrm{C}(\mathrm{n})$ & $\begin{array}{ll}\text { VWZ: } & 575^{\circ} \mathrm{C}\left(\mathrm{n}-25^{\circ} \mathrm{C}\right) \\
\mathrm{X}: & 525^{\circ} \mathrm{C}\left(\mathrm{n}-25^{\circ} \mathrm{C}\right)\end{array}$ \\
\hline $\mathrm{n} n$ & $850^{\circ} \mathrm{C}(\mathrm{n})$ & $850^{\circ} \mathrm{C}(\mathrm{n})$ & $\begin{array}{ll}\text { VWZ: } & 600^{\circ} \mathrm{C}(\mathrm{n}) \\
\mathrm{X}: & 550^{\circ} \mathrm{C}(\mathrm{n}) \\
\end{array}$ \\
\hline $\mathrm{n}+$ & $850^{\circ} \mathrm{C}(\mathrm{n})$ & $850^{\circ} \mathrm{C}(\mathrm{n})$ & $\begin{array}{ll}\text { VWZ: } & 625^{\circ} \mathrm{C}\left(\mathrm{n}+25^{\circ} \mathrm{C}\right) \\
\mathrm{X}: & 575^{\circ} \mathrm{C}\left(\mathrm{n}+25^{\circ} \mathrm{C}\right)\end{array}$ \\
\hline++ & $850^{\circ} \mathrm{C}(\mathrm{n})$ & $875^{\circ} \mathrm{C}\left(\mathrm{n}+25^{\circ} \mathrm{C}\right)$ & $\begin{array}{ll}\text { VWZ: } & 625^{\circ} \mathrm{C}\left(\mathrm{n}+25^{\circ} \mathrm{C}\right) \\
\mathrm{X}: & 575^{\circ} \mathrm{C}\left(\mathrm{n}+25^{\circ} \mathrm{C}\right) \\
\end{array}$ \\
\hline
\end{tabular}




\section{As-fired $10 \mathrm{k} \Omega$ - spread of values}

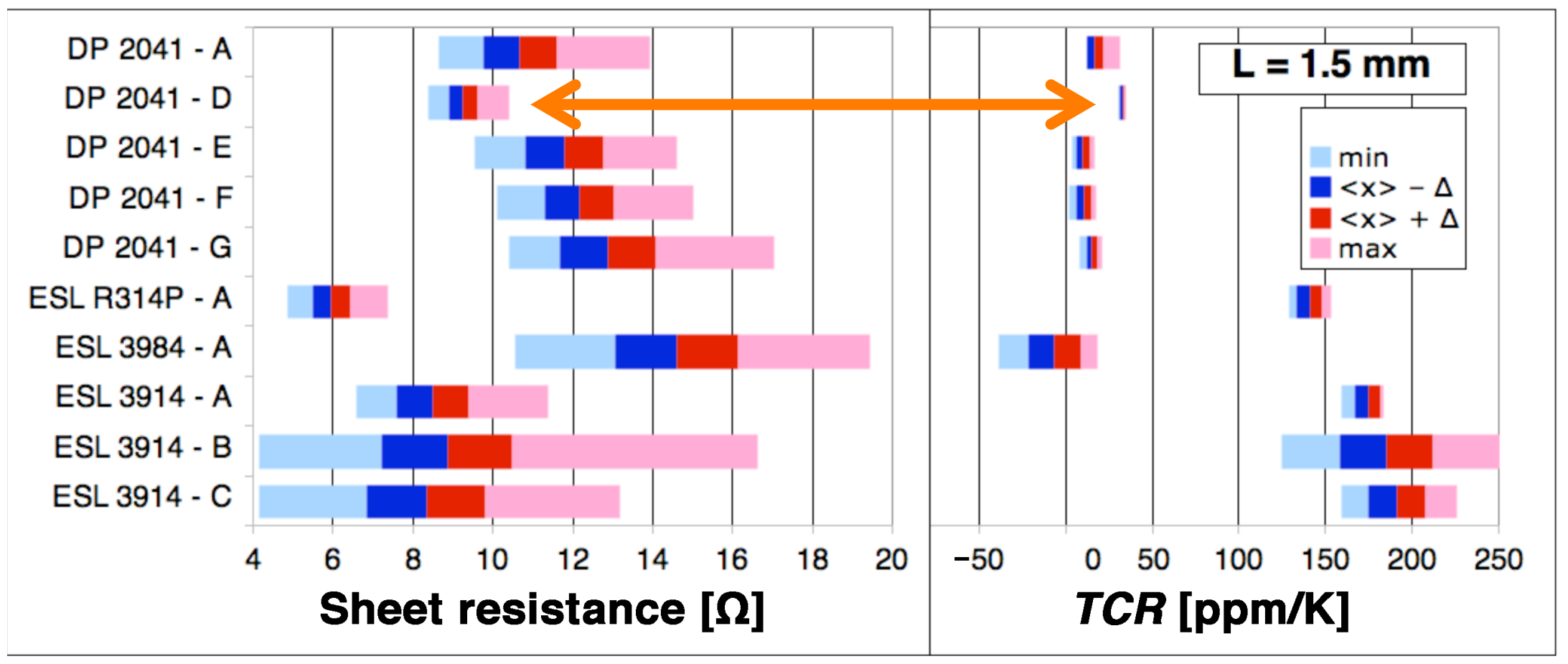

- Newer resistor compositions (DP 2041 / R314P) better

- Thin Au (D) terminations = lowest spread

- Low geometric disturbance of screen printing

- Low diffusion with terminations 


\section{As-fired $100 \Omega-$ spread of values}

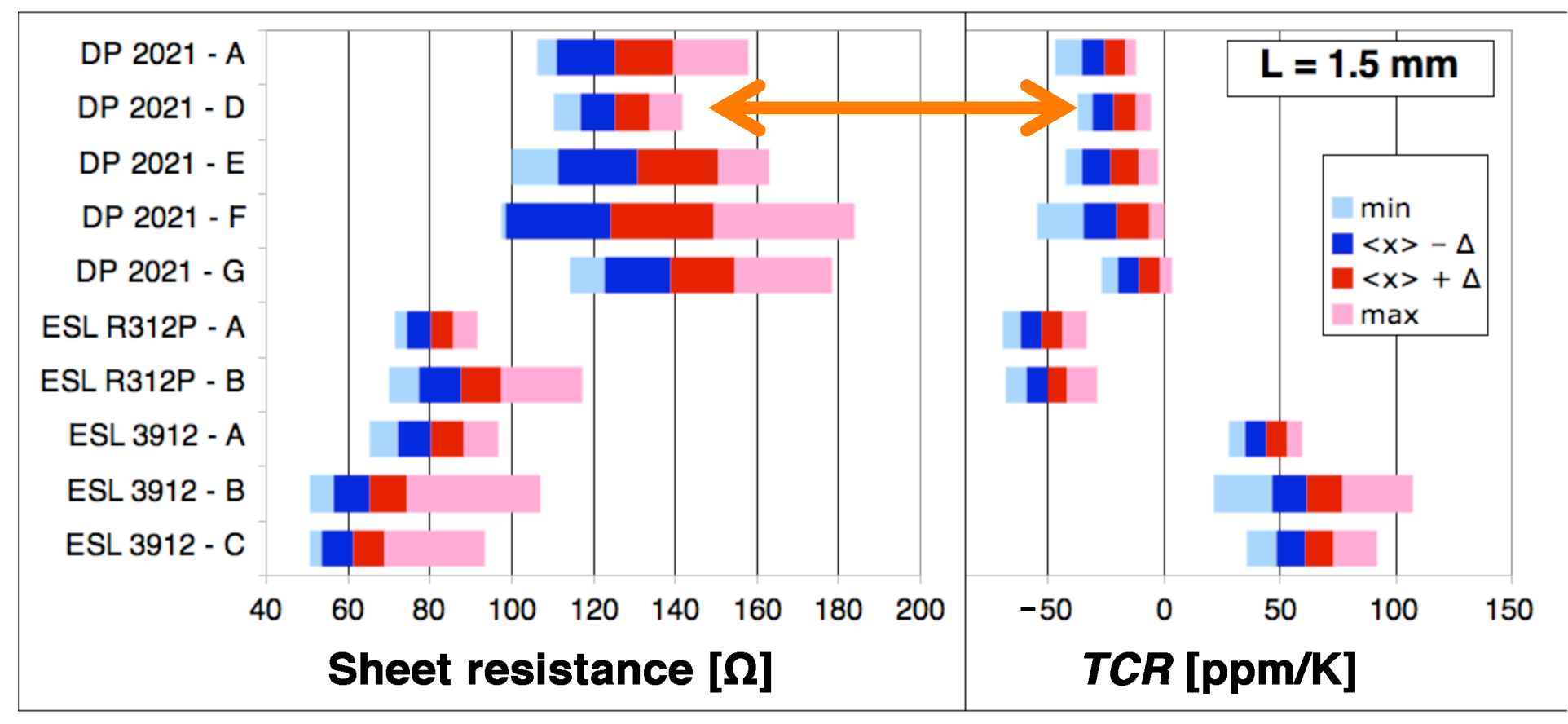

- Less difference seen in $100 \Omega$ compositions

- Not dominant - used for fine trimming 


\section{As-fired $10 \mathrm{k} \Omega$ - effect of process}
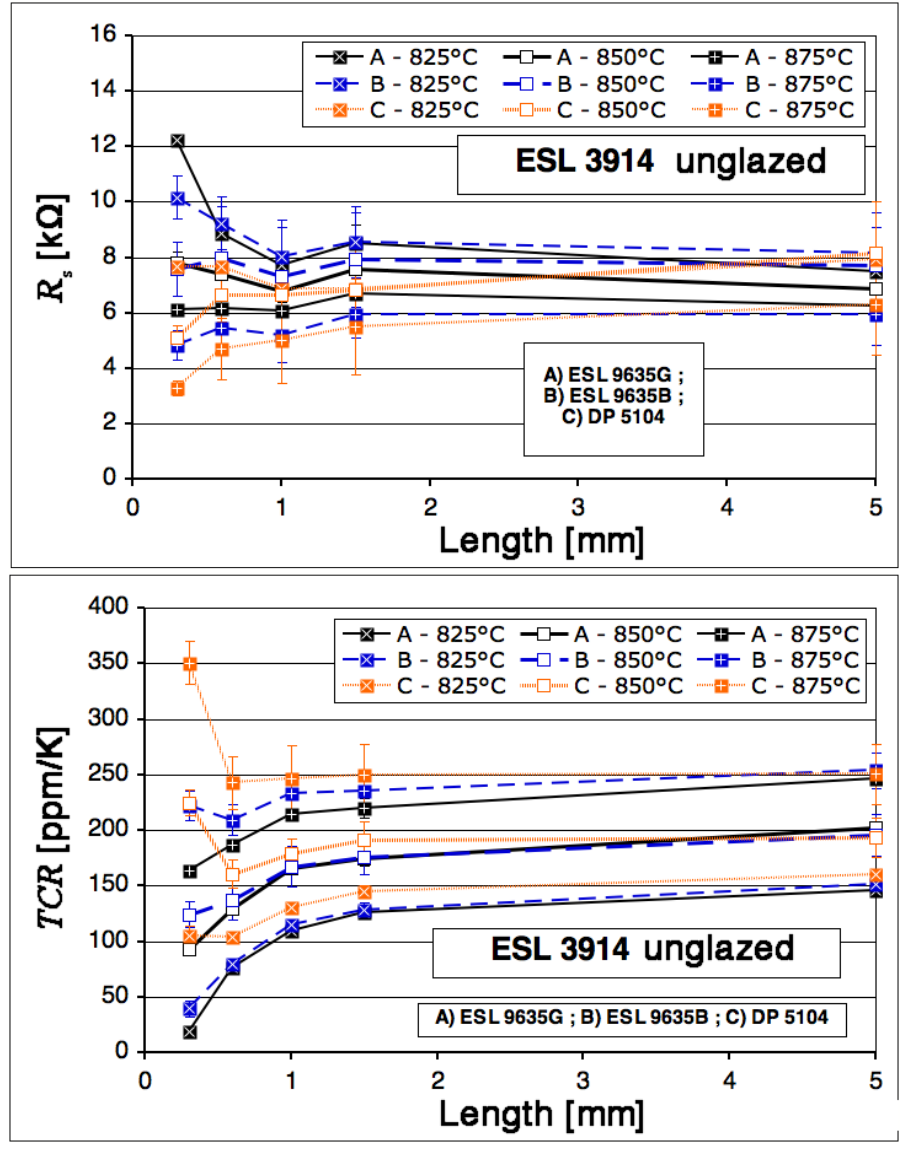
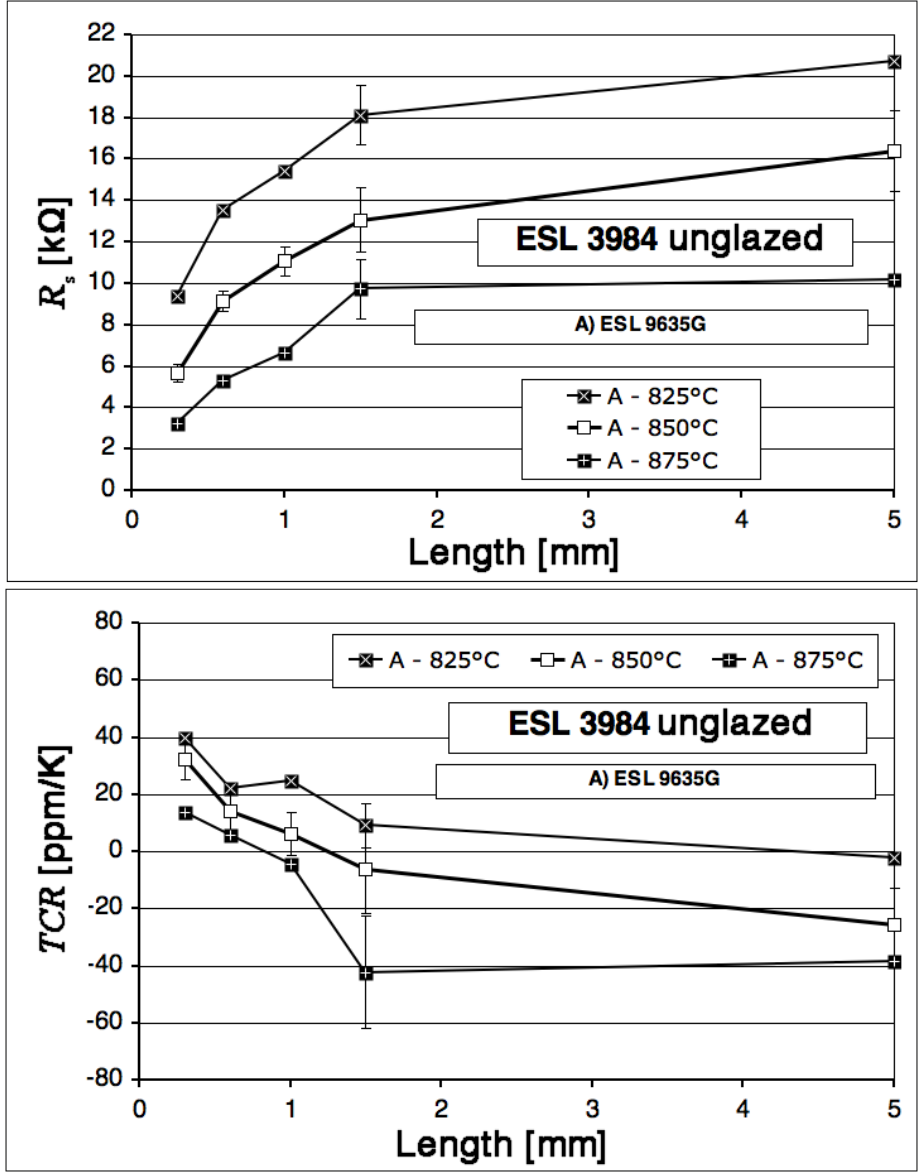

- Process dependence of value \& TCR different

- Strong length effects on TCR $->$ TCO for short resistors 


\section{Outline}

1. Introduction - manufacturing \& trimming issues

2. Resistor study

3. Overglazing, trimming, etc.

4. Conclusions \& outlook 


\section{Overglazing resistors}
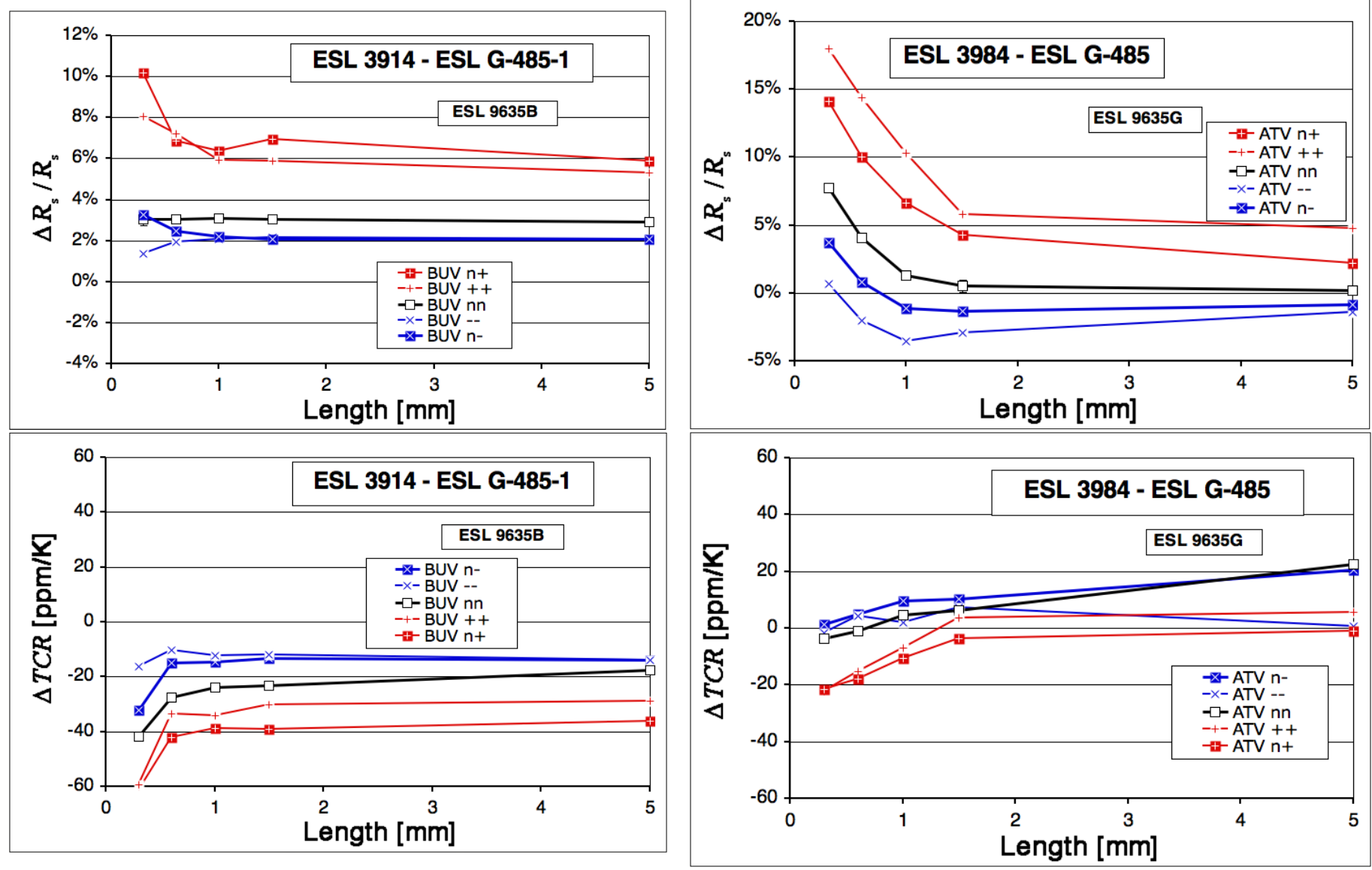

- Overglazing above nominal temperature - : strong drift

- Length dependence on $\triangle \mathrm{TCR}$ : leads to TCO 


\section{Trimming problems}
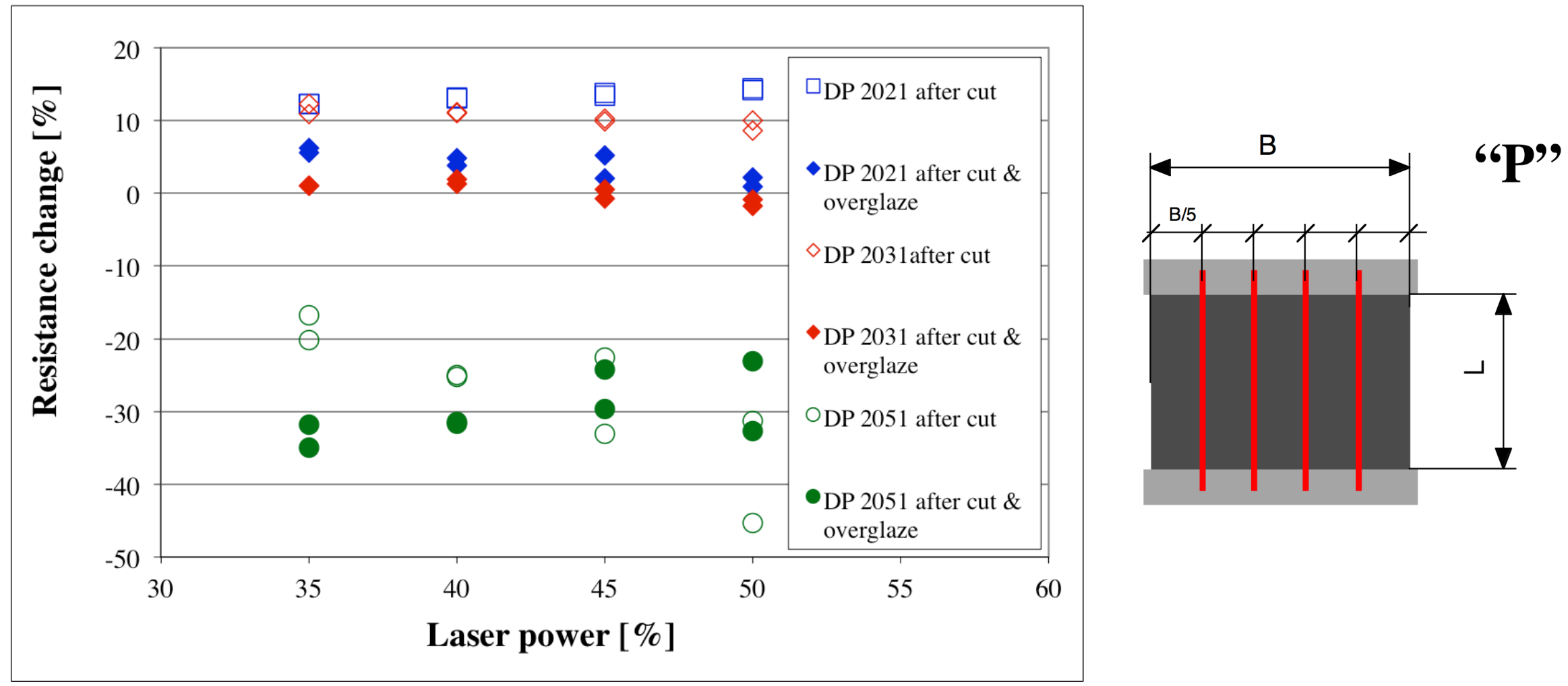

- Behaviour mostly normal: slight value increase

- Decrease of value for $100 \mathrm{k} \Omega$ composition! 


\section{Trimming \& stability of DP 2041 bridges}
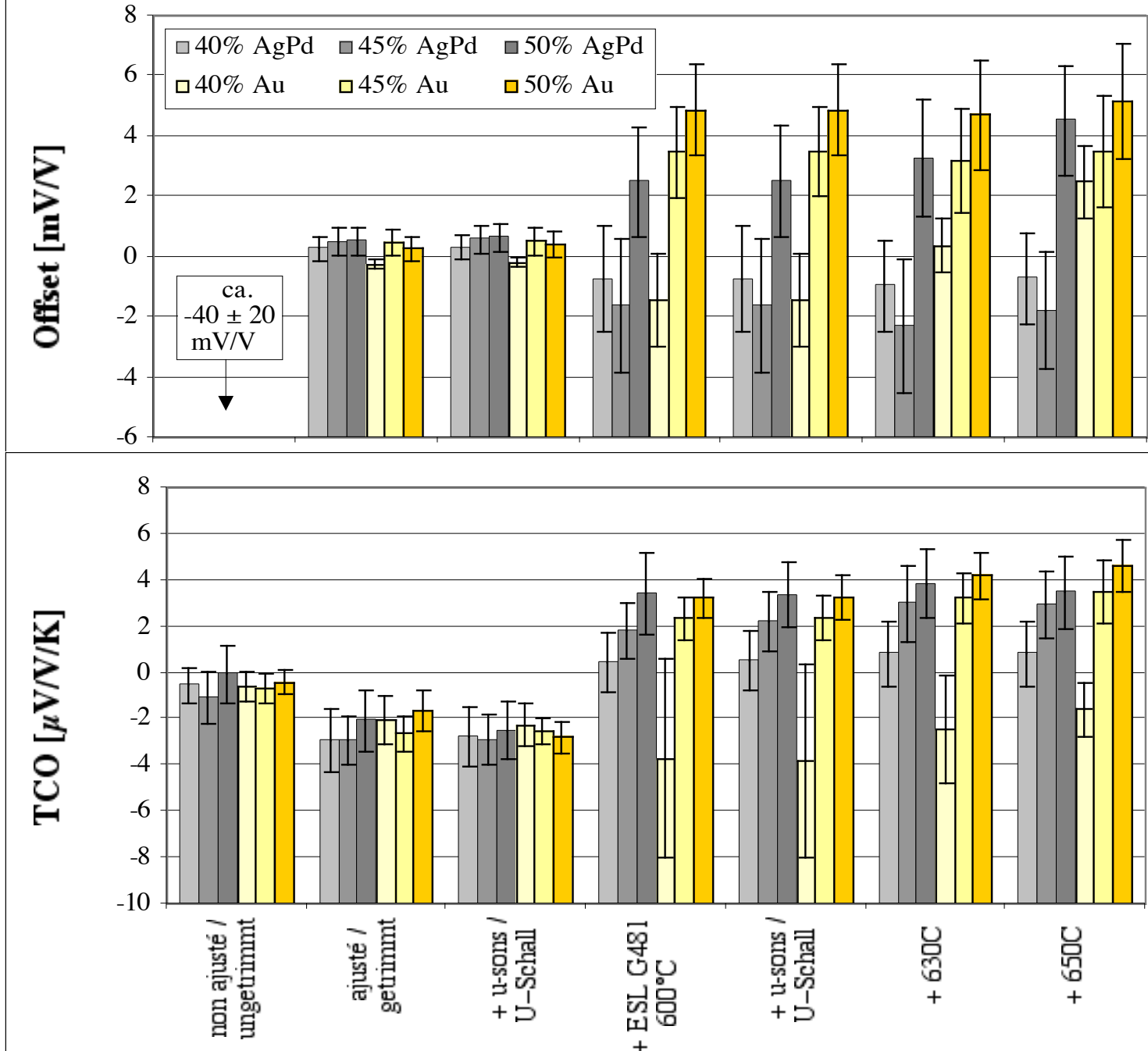

- Au initially $\sim 2 \times$ better than Ag:Pd

- After trimming

- Trim + ultrasound

- Advantage lost upon overglazing

- Trim-overglaze interactions dominant

- Temperature not so dominant (anneals)

- Better: refire overglaze or glaze again 


\section{Outline}

1. Introduction - manufacturing \& trimming issues

2. Resistor study

3. Overglazing, trimming, etc.

4. Conclusions \& outlook 


\section{Conclusions \& outlook}

- Thick-film piezoresistive sensors \& laser trimming

- Relatively low signal + harsh environments: difficult

- High process temperatures $->$ materials interactions critical

- Few alternatives to laser trimming (voltage?) for large series (cost)

- Best stability: start with discrete coarse trims

- Parameter development can be tedious

- Must ensure access of beam to resistor (not always practical!)

- Software offset trimming

- $\mathrm{R}_{\mathrm{adj}}=$ same paste as bridge, long meander (value $\sim 10 \times$ bridge)

- Little to no effect on TCO (if DAC reasonably good)

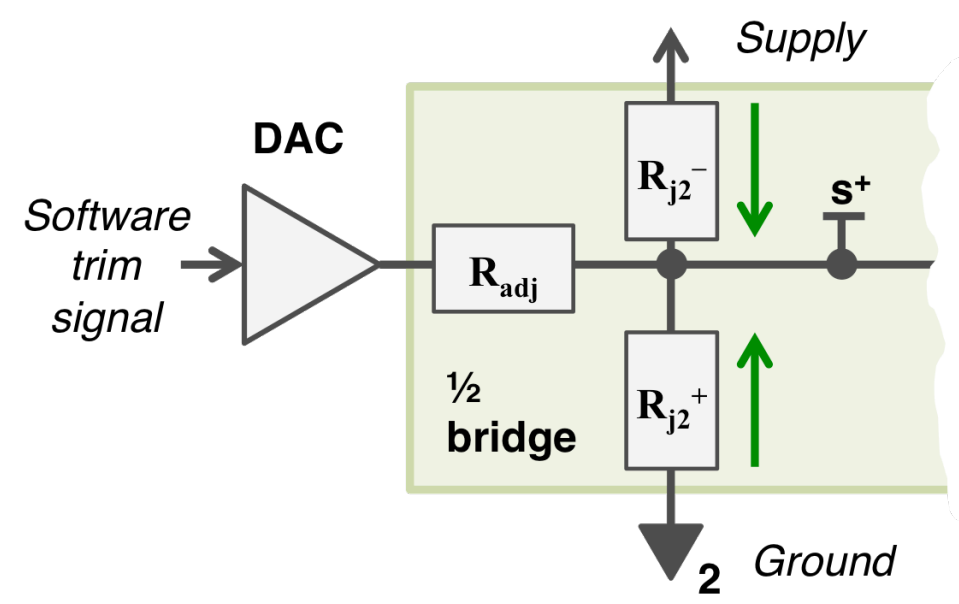




\section{Questions?}

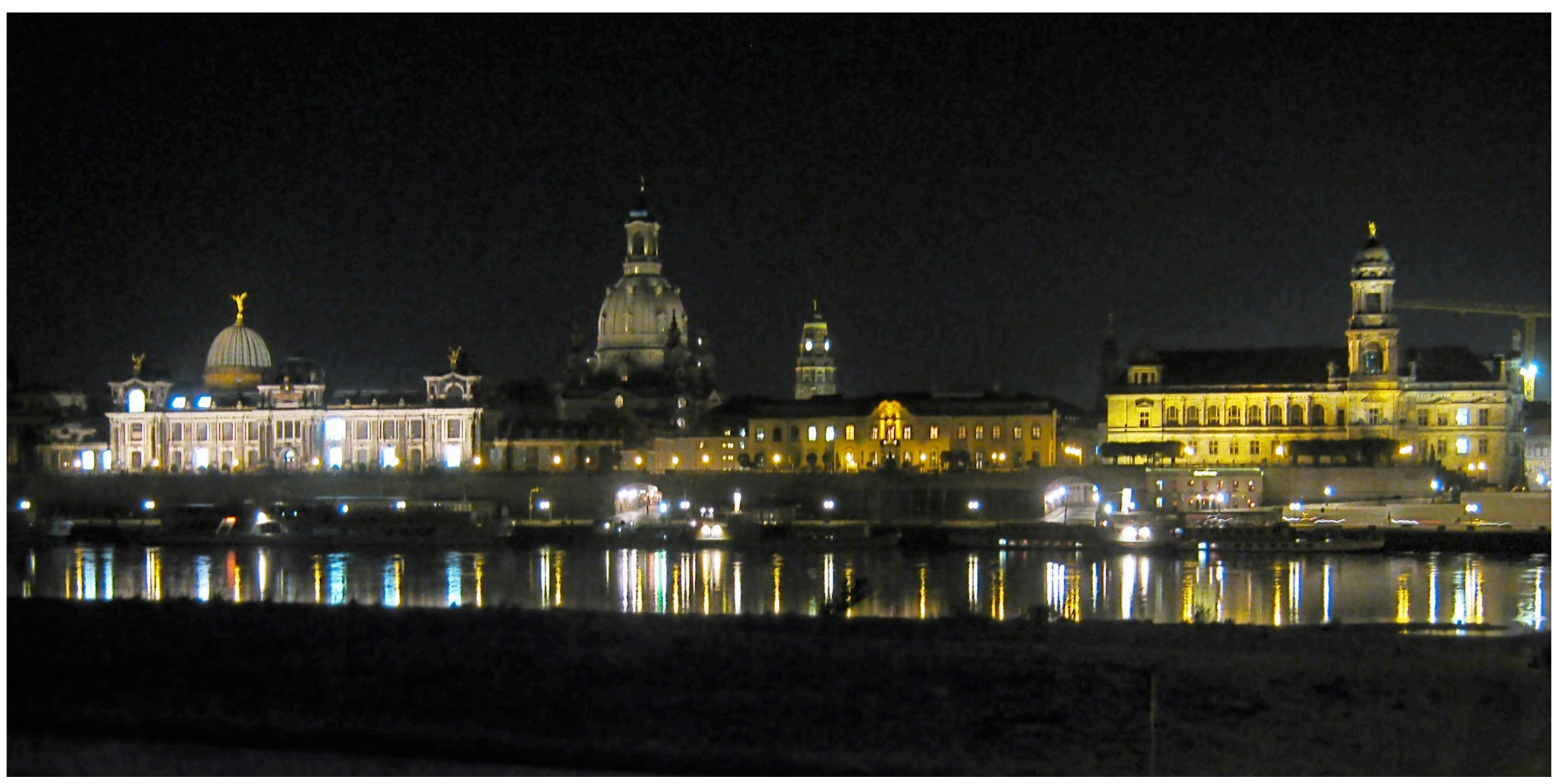

\section{THANK YOU!}




\section{Gauge factor measurement}

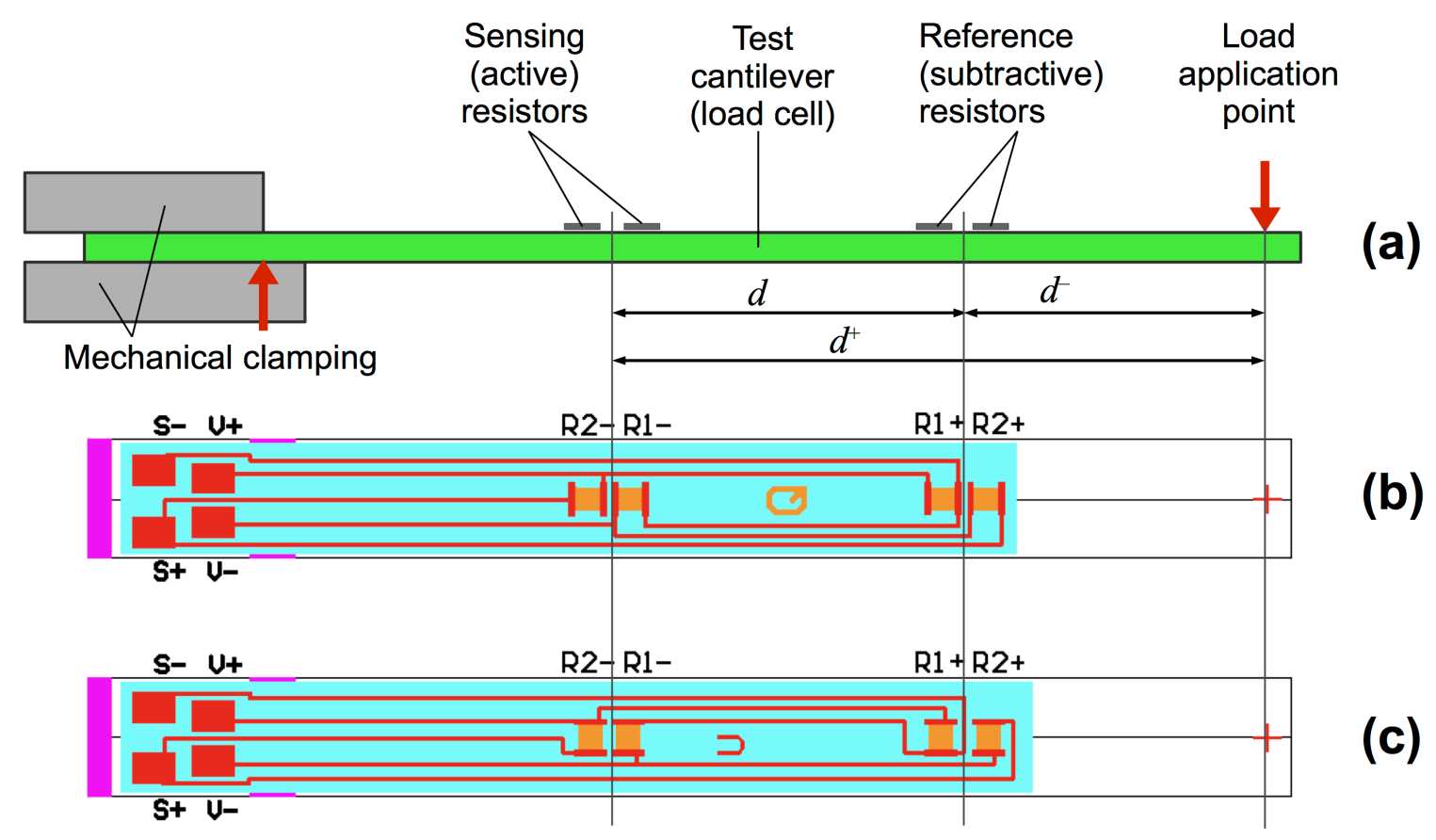

- Alumina cantilever

- Effective signal independent of loading errors 